\title{
Approaching Fake News at the Expense of Truth: A Psychophysiological Study of News on Social Media
}

\author{
Lauren E. Kirkwood \\ University of Hawai'i at Manoa \\ lkirkwoo@hawaii.edu
}

\author{
Randall K. Minas \\ University of Hawai'i at Manoa \\ rminas@hawaii.edu
}

\begin{abstract}
In 2018, sixty-eight percent of adults in America obtained their news from social media sites. During the same period, the amount of fake news online has increased substantially, resulting in increased propagation of false information. The research literature is growing on the effects of fake news on social media, but few studies have examined psychophysiological responses to true and fake news on social media. This research utilizes psychophysiological measures, specifically heart rate variability and skin conductance, to compare the perceived believability of news headlines posted on social media. Our findings indicate that individuals exhibit increased levels of approach behavior to true and fake news on social media. Additionally, higher time spent on social media is related to an increase in approach behavior to fake news. These findings have important implications for research and practice.
\end{abstract}

\section{Introduction}

The growth of social media (SM) over the last decade has connected society, creating social networks for people that span geographical and national boundaries. More recently, news stories have become integrated into SM sites, resulting in users being exposed to more information than ever before [27]. With this increase of news SM feeds, users need to think more critically than ever before. However, most SM users are in a hedonic state when they use SM and are less likely to think critically [7, 23]. This subsequently reduces the thoughtful interaction with the information and, instead of being more critical of the news story, SM users tend to view it as another form of entertainment [4]. In a similar vein, research has documented that sensationalizing news, purposely making news stories appeal to one's emotions, increases consumption of news $[12,33]$.
In the not too distant past, society obtained their information from printed newspapers that went through a series of required checks before widespread distribution. As of 2018, 68\% of adults in America retrieve news from SM sites [21]. Furthermore, 50\% of all Facebook users obtain news solely from Facebook, rather than taking multiple news sources into account. SM use has increased as well with the average person being on SM 2 hours and 22 minutes each day. News on SM continues to increase, and the rapid dissemination of fake news articles is poised to get worse [26].

Several research streams have focused on the "dark side" of SM use. Research has elucidated users of SM experience more loneliness, despite being more connected [13, 20]. Research has also shown SM enjoyment can eventually lead to maladaptive behaviors and psychological dependency [31] with research indicating neurological similarities with gambling and substance addiction [32]. Similarly, research has shown that increased use of SM can lead to attenuated psychophysiological responses [35].

Relatively few studies have examined psychophysiology of true and fake news on SM. One study has examined the neurophysiological correlates of fake news on SM, showing that propagation of fake news stories is driven by confirmation bias [11, 23]. However, these studies did not examine the emotional responses generated by true and fake news online. Given abundant literature on emotional appeals in traditional news, it is essential to study the role of emotions in news processed through SM.

This study examines the psychophysiological correlates of true and fake news online. We use theory from psychology and communications research to examine how an individual's appetitive and aversive motivational systems respond to true and fake news stories when the participant correctly identifies true and fake news and, perhaps more importantly, when the participant believes a fake article. We also examine the effect of time spent on SM. Our findings show fake news creates a larger emotional response than true 
news and relates to the amount of time people spend on SM. These findings have several implications for both theory and practice.

\section{Prior Theory and Hypotheses}

\subsection{Motivated message processing}

Human behavior is driven by two motivational systems. The appetitive system (which drives the approach response) and the aversive system (which drives the avoidance response) [14]. Humans have 2 limited cognitive resources that must be allocated to many stimuli present in the perceptual space. The limited capacity model of motivated mediated message processing (LC4MP) explains these activation patterns and the underlying psychophysiological responses of these systems in relation to media messages [14]. Theories propose that cognitive resources are allocated purposefully [eg., 15] — based on personal salienceand reflexively due to evolved mechanisms of information intake such as the orienting response [16, $22,25]$.

The appetitive and aversive systems can be activated individually (e.g., "liking" a post of a loved one's children or pets or scrolling past a post without engaging it) or they can become coactivated (e.g., engaging or "approaching" a post with a "sad" emoji). Individual activation of the appetitive system is associated with feelings of joy or happiness, while activation of the aversive system is associated with sadness or fear [18]. Coactivation is associated with a feeling of mixed emotion [18].

2.1.1. Psychophysiological measurement of the motivational systems. Measurement of the activation of the LC4MP motivational systems can be done through psychophysiological methods. Skin conductance is a measure of electrodermal activity that has been used for over a century. Changes in electrodermal activity are related to specific emotional and cognitive states. Increased electrodermal activity indicates an appetitive response, and has long been linked to emotion, arousal, and attention [5]. In this study, we used tonic skin conductance level (SCL) to indicate the average level of skin conductance over the 15 seconds that a subject viewed a news headline.

The relationship between heart rate and sympathetic or parasympathetic nervous system activation is more complex. Respiratory sinus arrythmia (RSA) is defined as the change in heart period corresponding with the inspiratory and expiratory phases of the respiratory cycle [28]. It is closely related to the high frequency $(0.15-0.40 \mathrm{~Hz})$ component of heart rate variability. RSA reflects the vagal cardiac influence, mediated by the vagus cranial nerve, and is highly responsive to stimuli present in our environment [3].

While skin conductance measures appetitive system activation, heart rate can indicate either appetitive or aversive systems. Increases in RSA indicate increased activation of the aversive motivational system, while decreases in RSA indicate activation of the appetitive motivational system.

\subsection{Fake news and social media}

2.2.1. Spread of fake news on social media. Fake news and its dissemination on SM came to the attention of the public around the 2016 Presidential Election in the United States. Since 2016, there has been increasing concern over the spread of fake news through SM outlets. Many of the fake news articles posted on SM sites believed, which leads propagation of false information [34]. Research has indicated that fake news articles travel faster than true news stories [34]. This study analyzed 126,000 stories posted on Twitter from 2006 to 2017 and found the fake stories reached 1,000 to 100,000 people whereas the true stories rarely reached more than 1,000 people [34].

It is also important to note that the format of the information has an effect on the level of perceived credibility of the information [29]. People tend to be more likely to believe information when it is formatted like a news story rather than portrayed as a story. Additionally, confirmation bias exacerbates the problem, as people are more likely to believe information that aligns with their opinion than information that contradicts it [29]. It has also been shown that presenting source ratings directly affects the believability of an article, so this could be one way to influence the readers to evaluate the information more critically [29].

The effects of the propagation of false information can be far reaching, as individuals anchor on a priori beliefs in the face of evidence that directly contradicts it [23]. Upon learning that information initially thought to be true was actually false, people do tend to adjust their attitudes, but to varying levels [30]. Therefore, if an article is advertised as true, and is clearly refuted as false, a portion of society will continue to believe the original, false information. This discovery makes the filtering of information posted on SM even more crucial to the political and social health of society.

Understanding how appetitive and aversive responses relate to fake news can help determine how it propagates on SM [34]. Studies that have applied LC4MP to SM have found appetitive responses to positive and negative messages [1]. Other studies have 
found that individuals are more likely to engage with negative information on Twitter [10]. These results on emotional valence or arousal but did not factor in an individual's a priori beliefs. Past literature has shown differential processing of information according to beliefs both on SM and in collaboration [22, 23]. An individual's confirmation bias would create an approach response for articles that conform with an individual's prior beliefs. Furthermore, individuals tend to not process information that is contrary to prior beliefs [22] and are likely to exhibit a aversive response to information they do not believe. Therefore, we hypothesize:

H1: An individual's appetitive system will be activated when they (a) believe a true article on social media and (b) believe a fake article on social media.

$\mathrm{H} 2$ : An individual's appetitive system will be activated when they (a) do not believe a true article on social media and (b) do not believe a fake article on social media.

2.2.2. Time spent on social media. Research into SM use and its effect on emotion has provided mixed findings. Previous research on the amount of time spent on SM and loneliness found a significant relationship between increased use and feelings of isolation [2]. These findings, however, were later found to be caused by a confounding variable of social anxiety [6]. A study on how Facebook use relates to self-esteem and college adjustment found that spending a time on Facebook is related to low self-esteem, and the number of Facebook friends was negatively associated with emotional adjustment for first-year undergraduate students [9]. Furthermore, multitasking with media has been found to have an association with higher depression and social anxiety symptoms [2].

Research has also studied SM addiction, as it has been found that increased enjoyment while using SM also has the potential adverse effect of addiction. Excessive SM use, especially habitual use characteristic of dependency, has potentially damaging psychological, physiological, and social effects [31]. Research has found SM addiction affects the brain similarly to drug or gambling addictions [32].

In terms of psychophysiological responses to SM, prior research has shown that sympathetic arousal diminishes over time [35]. As a user spends more time on Facebook, their physiological response lessens, throughout the specific session and potentially with SM use in general.

There is a paucity of research using psychophysiology to study fake news on SM. One forthcoming study has used neurophysiology to understand the effect of flagging false news on SM and confirmation bias [23]. This study showed specific cognitive patterns of confirmation bias and cognitive dissonance when individuals interacted with fake news on SM [23]. This study suggests that SM use is associated with increased activation of the frontal cortices while processing two discordant pieces of information (i.e., an individual believes an article flagged as fake). This study could provide support for coactivation of the motivational systems as SM use increases.

Individuals that use SM regularly may approach information differently than those that use SM only occasionally. The increased use of SM is driven by the appetitive system. However, some interactions on SM may lead to both the appetitive and aversive systems becoming active. Thus, we hypothesize:

H3: For heavy social media users, coactivation will occur when viewing true and fake articles on social media.

H4: For heavy social media users, correctly identifying a news article as true or false will activate the appetitive system.

H5: For heavy social media users, incorrectly identifying a news article as true or false will result in coactivation of the motivational systems.

Past research has established a connection between the appetitive motivational system and a desire to learn from features of news articles [24]. Similarly, social information seeking, or wanting to keep abreast of the statuses of people on social media has also been linked to activation of the appetitive system [35]. In this study, we hypothesize that the more an individual wish to stay informed of news on SM, the greater their appetitive response:

H6: Individuals that wish to stay informed on current events will display activation of the appetitive system when viewing true and fake articles on social media.

2.2.3. Reposting true and fake news. Research has shown that SM likes and shares occur most often when there is an appetitive response to the post, followed by coactive responses, and aversive [1]. However, this finding has not been applied to fake news on SM. The same appetitive response would be expected when wanting to repost an article or post an article with an opinion. Furthermore, time spent on SM should increase the appetitive response and result in an increased likelihood to post and to post with an opinion. Thus, we hypothesize: 
H7: Individuals will experience increased activation of their appetitive system when they (a) intend to repost the story on social media and (b) intend to post an article with their opinion on social media

H8: Individuals that spend more time on social media will experience increased activation of their appetitive system when they (a) intend to repost the story on social media and (b) intend to post an article with their opinion on social media.

\section{Method}

\subsection{Participants}

Twenty-six students were recruited from computer science courses at a large state university in the U.S. Participants received extra credit for completing the study. Information on an individual's age and gender were collected via demographic questionnaire. Ages ranged from 18 to 44 with an average of 22.1. Nineteen participants $(73 \%)$ were male and seven participants were female (27\%).

\subsection{Procedures}

The participants arrived at the laboratory at individual times, and informed consent was obtained. The participant was then seated in a stable chair at a computer separated from the researcher by a partition. The desktop computer was equipped with the preloaded questionnaire in Qualtrics. After being seated the participants were given instructions on the experiment Two BIOPAC sensors were placed on the participant's left foot in the plantar configuration for skin conductance data collection. The participants then were instructed to begin the experiment. They filled out a brief demographics questionnaire.

The primary task in this study was viewing 50 images of news article headlines that had been posted online then rating them on follow-up dimensions. The participants viewed each image for 15 seconds, then were asked to assess the believability of the headline. Furthermore, participants were asked a series of follow-up questions on their intention to share the information directly on SM and their intention to share the information with their opinion.

After the questionnaire they began viewing and responding to the first set of 25 news article snapshots. The snapshots had been randomized so the first 25 images were a mix of true and false articles, and each participant viewed a different order. The stimulus (the news article headline, picture and short stub) appeared on the screen alone for 15 seconds, then the follow-up questions were automatically displayed on the screen at the end of the 15 seconds. The stimuli remained present on the screen during the follow-up questions about credibility and the participant's intent to share the article. Following the questions on how the article made them feel, a new stimulus was presented for 15 seconds. After 25 images had been viewed and rated, the participant completed a short personality questionnaire to as a break. After the questionnaire was complete, the participant viewed the next 25 true or false news article snapshots, again presented randomly. The experiment ended with a separate survey on handedness. Participants were also asked to answer questions about their use of SM and news consumption. The participants were then released.

\subsection{Treatments}

This study was a repeated measures experiment that used images of online newspaper article snapshots (Figure 1 and 2), which had each been rated as true or false by an independent, third-party fact-checker, Snopes.com. Snopes has grown to be the largest independent fact-checking site on the Internet. Twentyfive true online articles and 25 false online articles were chosen for this study. These articles were rated by Snopes as either clearly "true" or "false." Snopes also provides extensive documentation that illustrates why they have reached each conclusion. Snopes addresses articles that have received a level of public interest and have been widely reported in the media.

Once the 50 article snapshots were selected they were then randomized and split into two separate blocks within the questionnaire. The participants viewed each image of the online articles and rated each on perceived believability and their intent to share. Half-way through the experiment, after 25 images had been shown to the participant, a short questionnaire in the middle provided a break from rating the images. All behavioral responses were presented and collected using the experimental survey tool Qualtrics.

\subsection{Dependent variables}

The dependent variables in this study were appetitive and aversive activation, which was quantified using skin conductance obtained from EDA measurements and RSA derived from electrocardiogram (ECG) measurements. 


\section{EPA dismisses half of key board's scientific advisers; Interior suspends more than 200 advisory panels}

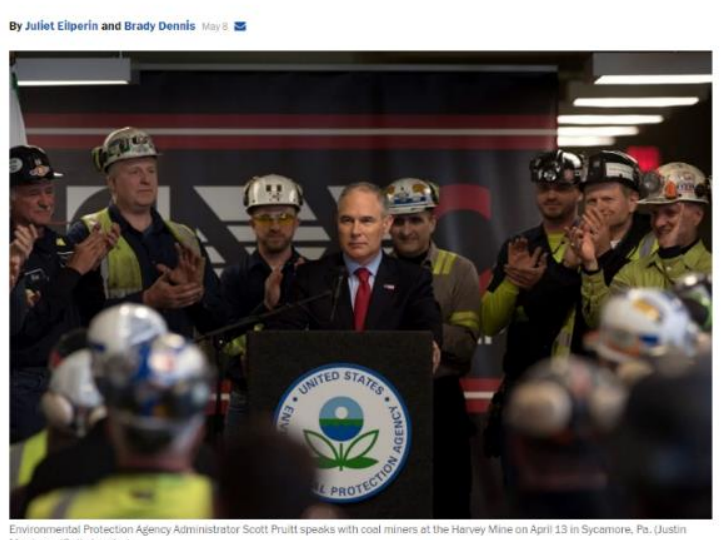

Figure 1. Example of article snapshot rated as "true" by Snopes.com

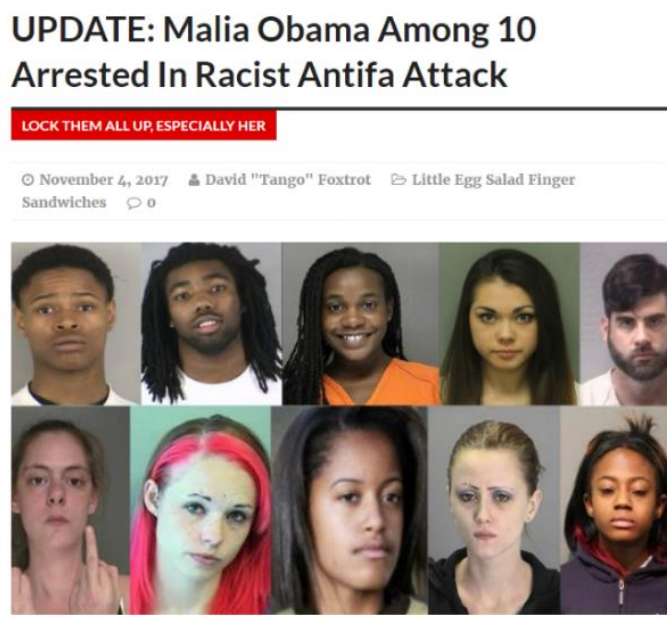

Join America's Last Line of Defense And Share This Article Now! f SHARE ON FACEBOOK Y SHARE ON TWITIER

Figure 2. Example of article snapshot rated as "false" by Snopes.com

Increases in skin conductance indicate appetitive system activation and further indicate the level of attention or excitation of the participant [19]. Skin conductance was measured by adhering two EDA $\mathrm{Ag} / \mathrm{AgCl}$ electrodes from BIOPAC Systems to the plantar surface of the left foot. The plantar surface was prepared with an alcohol wipe, then two electrodes pregelled with isotonic gel were adhered to the skin. The BIOPAC MP150 collected at $1000 \mathrm{~Hz}$.

RSA was obtained by collecting ECG data and deriving heart rate variability. Increases in RSA indicate increases in parasympathetic nervous system activation [29]. In this study ECG data was collected by adhering a BIOPAC disposable electrode to each arm. The $\mathrm{Ag} / \mathrm{AgCl}$ electrodes were pre-gelled with electrolytic gel and were placed directly on the surface of the skin after prepping the area with an alcohol wipe. The signal for both EDA and RSA were collected using Acqknowledge software version 4.4.0.

\subsection{Analysis}

Analyses were completed using Hierarchical Linear Modeling (HLM) version 6.08 on the dependent psychophysiological measures RSA and EDA. The independent within-subject variables were whether someone believed a true or fake article, did not believe a true or fake article, or whether the participant intended to share the post directly or with their opinion. The independent between-subject variables were self-reported SM use and desire to stay informed on current events. All variables were grand mean centered and robust standard errors were used in the model.

\section{Results}

Statistical results for the HLM model are provided in Table 1. A summary of our findings is provided in Figure 3 and Table 2.

A significant main effect was observed for RSA $(\mathrm{t}(1215)=2.074, \mathrm{p}=0.038)$ and EDA $(\mathrm{t}(1224)=$ $2.641, \mathrm{p}=0.009)$ when a subject believed an article that was true. These measures indicate that when an individual believes a true article there is coactivation of the appetitive and aversive systems. Thus, H1a is partially supported, the appetitive system is active. However, the aversive system is also activated.

A main effect was observed for EDA $(\mathrm{t}(1224)=$ $3.217, \mathrm{p}=0.002$ ) when a subject believed an article that was fake. This indicates that when an individual believes a false article, there is an appetitive response. $\mathrm{H} 1 \mathrm{~b}$ is supported.

No psychophysiological effect was observed when an individual mistakenly rejected a true article. Thus, $\mathrm{H} 2 \mathrm{a}$ was not supported. A main effect was observed for EDA $(\mathrm{t}(1224)=2.419, \mathrm{p}=0.016)$ when a subject correctly rejected a fake article. This indicates that when an individual does not believe a false article there is an increased appetitive response. This finding was contrary to $\mathrm{H} 2 \mathrm{~b}$ but indicates fake news creates an approach response.

A main effect of time spent on SM was observed for RSA $(\mathrm{t}(1215)=7.043, \mathrm{p}<0.001)$ and EDA $(\mathrm{t}(1224)=3.785, \mathrm{p}<0.001)$. These measures indicate coactivation of the appetitive and aversive systems for 
individuals that spend more time on SM. H3 was supported.

Time spent on social media did not have an effect on the appetitive or aversive systems when an individual believed a true article. However, an interaction effect was observed for RSA $(\mathrm{t}(1215)=$ $1.979, \mathrm{p}=0.048$ ) with individuals that spend more time on SM having a significant aversive response when they do not believe a fake article. Therefore, $\mathrm{H} 4$ was partially supported.

An interaction effect was also observed for RSA $(\mathrm{t}(1215)=-2.029, \mathrm{p}=0.042)$ for individuals that spend more time on SM. This measure indicates an appetitive response for individuals that spend more time on SM when they believe a false article. Individuals had an appetitive response to fake articles they believed. No significant effect was observed on individuals that spend time on SM and reject true article. Thus, H5 was partially supported.

A main effect was also observed for individuals that like to stay informed in RSA $(\mathrm{t}(1215)=-2.939$, $\mathrm{p}$ $=0.004)$ and EDA $(\mathrm{t}(1224)=6.028, \mathrm{p}<.001)$. Both measures indicate increased appetitive activation for individuals that like to stay informed of current events compared to individuals that do not. Thus, H6 is supported.

A main effect was observed for RSA $(\mathrm{t}(1215)=$ $2.924, \mathrm{p}=0.004$ ) when a subject intended to share an article and also add their opinion on SM. This measure indicates an appetitive response when an individual intends to share the article with their opinion. Thus, $\mathrm{H} 7 \mathrm{~b}$ is supported. An interaction effect was also observed for EDA $(\mathrm{t}(1224)=3.638, \mathrm{p}<.001)$ between individuals who spend more time on SM and the intent to share the article with their opinion. The EDA measure indicates a significant appetitive response. $\mathrm{H} 8 \mathrm{~b}$ is supported.

\section{Discussion}

This research adds to a growing body of literature on true and fake news spread on SM. It uses LC4MP theory to better understand emotional reaction to news on social media and the appetitive and aversive responses to it. Our findings provide psychophysiological evidence of how individuals respond to true and false news when they believe or disbelieve it. These findings have several implications for research.

\subsection{Implications for research}

This study makes four core contributions to the literature. First, fake news created a more appetitive response than true news that was either believed or not believed. Fake news that was believed elicited an approach response, while true news that was believed created mixed emotions or ambivalence to the article.

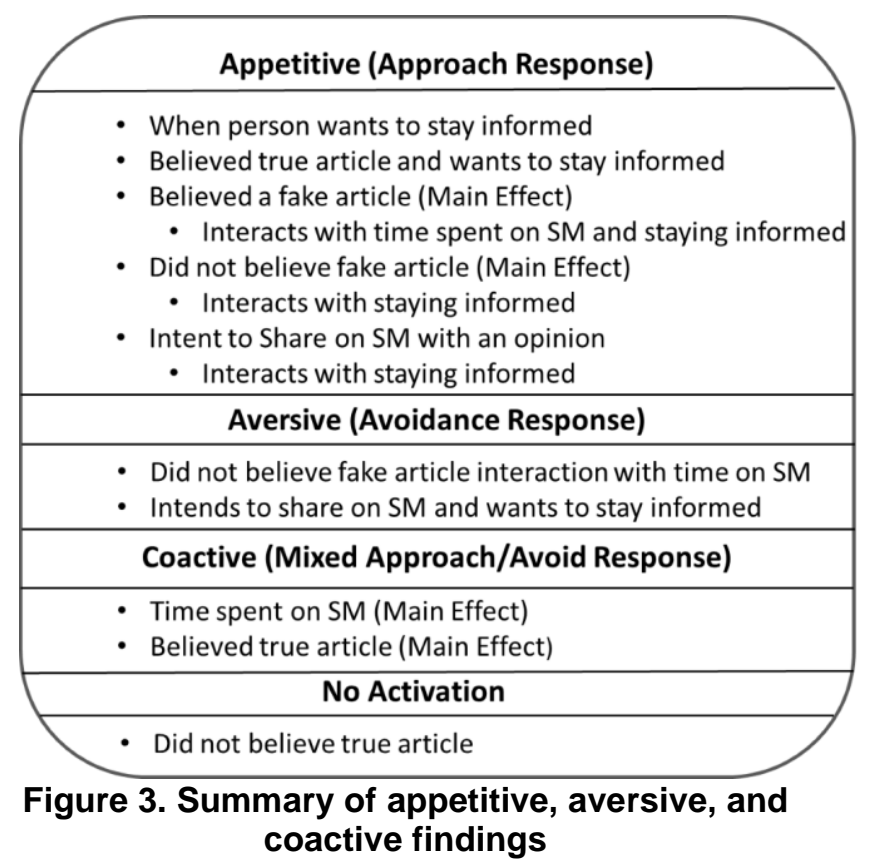

\section{Table 2. Summary of interactions}

\begin{tabular}{|c|c|c|}
\hline & True News & Fake News \\
\hline $\begin{array}{l}\text { Time Spent on } \\
\text { SM }>75 \text { th } \\
\text { Percentile }\end{array}$ & No Effect & $\begin{array}{l}\text { Increased } \\
\text { psychophysiological } \\
\text { response }\end{array}$ \\
\hline $\begin{array}{l}\text { Staying Informed } \\
>75^{\text {th }} \text { Percentile }\end{array}$ & $\begin{array}{l}\text { Increased } \\
\text { psychophysiological } \\
\text { response when they } \\
\text { believe it }\end{array}$ & $\begin{array}{l}\text { Increased } \\
\text { psychophysiological } \\
\text { response }\end{array}$ \\
\hline
\end{tabular}

Second, we observed a main effect of increased coactivation of the appetitive and aversive systems that related to the amount of time spent on SM. This result suggests increased use of SM is associated with mixed emotions when encountering news articles. When examining error rates in believing true and false news, an interesting finding emerged. Individuals approach fake news with an approach response as they spend more time on SM. Taken with prior research on confirmation bias on SM [11, 23], this indicates that the confirmation bias is present when you believe a fake news article and is also influenced by the degree to which you use SM. The more you use SM, the higher your approach response to fake news you believe.

Similarly, individuals that use SM more often have a larger aversive response to fake news articles they do 
not believe. Furthermore, individuals have attenuated responses to true information they do not believe, which aligns with prior research on confirmation bias in SM (i.e., if they do not believe it, the motivational systems do not respond to it).

The coactivation finding with increased SM use needs to further research as well. This suggests, the more individual's SM, the greater mixed emotions individuals have. These findings fall in line with previous research on long-term effects of SM use and addiction. While our finding did not indicate addiction, it does indicate increased SM use is associated psychophysiology for skin conductance and heart rate. A longitudinal study could elucidate which is the causal factor of these psychophysiological changes.

Our third contribution is how an individual's desire to stay informed on current events affects their appetitive and aversive responses to believing true and fake news. The greater the individual's desire to stay informed, the higher their appetitive response to all news articles. More importantly, individuals that like to stay informed on current events have an increased appetitive response to fake news they believe and an appetitive response to fake news they do not believe. They also exhibit an appetitive response to true articles they believe but have no reaction when they do not believe a true article. The implication of this finding is that individuals that like to stay informed on current events are susceptible to engaging fake news even more than true news they do not believe. Fake news elicits an emotional response, while only true news they believe elicits an emotional response.

Our fourth contribution is on intent to share directly on SM or to share with the addition of their opinion. The more individuals like to stay informed, the more aversive their response is to sharing articles directly. Further when individuals intend to share an article and add their opinion, the greater the activation of the appetitive system. However, this effect is more pronounced for individuals that spend more time on SM.

Taken together, these results extend our understanding of true and false news on social media by elucidating the motivational factors underlying how individual's approach or avoid processing news articles on SM. These findings further extend the work on confirmation bias and dissonance on SM by showing the motivational underpinnings of appetitive or aversive motivation that exist downstream of the highlevel cognitive processes. They further indicate fake news is better at eliciting emotional reactions from individuals than true news. This effect on emotion is compounded by the time an individual spends on SM and how much the individual likes to stay informed on current events. In the next section, we discuss implications for practice based on these findings.

\subsection{Implications for practice}

This study underscores the importance of thirdparty fact-checking. SM companies need to provide more tools to combat confirmation bias, which leads to increased processing of fake news. In addition, individuals should be aware of the implications of only getting their news on SM. Individuals need to click through to the articles to help better determine true and false information online. It seems problematic for news to be shared on SM in general as users are in a hedonic state, bring their own biases, and are insulated by an algorithmic "bubble" of what the platform believes they want to see.

\subsection{Limitations and future research}

This research suffers from the usual limitations of laboratory research: we studied student participants who worked on two short-duration tasks which had no intrinsic value to them. Much research on social media has used laboratory studies with student subjects because the use of a laboratory experiment with student subjects was appropriate for testing the theory, given the desire for the tight control that laboratory experiments provide [8, 17]. As prior experience with any technology often influences performance, we used a repeated measures design to better control for differences among individual backgrounds and experience.

The sample size $(n=26)$ was low for some of our between subjects measures, namely differentiating between low and high social media use and desire to stay informed on current events, the within-subjects measures are robust due to the sampling rate of the psychophysiological measures and increased statistical power of the repeated measures design.

In this study, there were a number of hypotheses that were not confirmed, or the alternate hypotheses was accepted. This study sought to integrate the LC4MP theory with the emergent topic of fake news on social media. The hypotheses that were not confirmed, or the instances where a competing hypothesis was suggested, warrant further examination. An extension of this research and further elucidation of the relationship between the appetitive and aversive systems is needed in light of the current findings. Future research should also confirm the hypotheses that were supported in this research. 


\section{Conclusion}

This study adds to the growing body of literature of increased information processing of fake news over true news. The study provided psychophysiological evidence that fake news is related to approach behavior more so than true news, resulting in higher skin conductance and changes in heart rate variability. This study provides further evidence that increased social media use has an effect on individuals, resulting in a stronger appetitive response to fake news than low levels of social media use. The results of this study provide a clearer picture of why fake news spreads quickly online, underscoring the need for further research in this area.

\section{References}

[1] S. Alhabash, A.R. McAlister, A. Hagerstrom, E.T. Quilliam, N.J. Rifon, and J.I. Richards, "Between Likes and Shares: Effects of Emotional Appeal and Virality on the Persuasiveness of Anticyberbullying Messages on Facebook", Cyberpsychology, Behavior, and Social Networking, 16, 3, 2013, pp. 175-182.

[2] M.W. Becker, R. Alzahabi, and C.J. Hopwood, "Media Multitasking Is Associated with Symptoms of Depression and Social Anxiety", Cyberpsychology, Behavior, and Social Networking, 16, 2, 2012, pp. 132-135.

[3] G.G. Berntson, J. Thomas Bigger Jr., D.L. Eckberg, P. Grossman, P.G. Kaufmann, M. Malik, H.N. Nagaraja, S.W. Porges, J.P. Saul, P.H. Stone, and M.W. Van Der Molen, "Heart rate variability: Origins, methods, and interpretive caveats", Psychophysiology, 34, 6, 1997, pp. 623-648.

[4] P. Boczkowski, E. Mitchelstein, and M. Matassi. "Incidental news: How young people consume news on social media". in Proceedings of the 50th Hawaii International Conference on System Sciences. 2017.

[5] J.T. Cacioppo, L.G. Tassinary, and G. Berntson, Handbook of psychophysiology, Cambridge University Press, 2007.

[6] S.E. Caplan, "Relations Among Loneliness, Social Anxiety, and Problematic Internet Use", CyberPsychology \& Behavior, 10, 2, 2007, pp. 234242. [7] K. Chauhan and A. Pillai, "Role of content strategy in social media brand communities: a case of higher education institutes in India", Journal of Product \& Brand Management, 22, 1, 2013, pp. 40-51.

[8] D. Compeau, B. Marcolin, H. Kelley, and C. Higgins, "Research commentary - Generalizability of information systems research using student subjects-A reflection on our practices and recommendations for future research", Information Systems Research, 23, 4, 2012, pp. 1093-1109.
[9] M. Kalpidou, D. Costin, and J. Morris, "The Relationship Between Facebook and the Well-Being of Undergraduate College Students", Cyberpsychology, Behavior, and Social Networking, 14, 4, 2010, pp. 183-189.

[10] J. Kätsyri, T. Kinnunen, K. Kusumoto, P. Oittinen, and N. Ravaja, "Negativity Bias in Media Multitasking: The Effects of Negative Social Media Messages on Attention to Television News Broadcasts", PLOS ONE, 11, 5, 2016, pp. e0153712.

[11] A. Kim and A.R. Dennis, "Says Who? How News Presentation Format Influences Believability and the Engagement of Social Media Users", MIS Quarterly, in press.

[12] H.J. Kim and G.T. Cameron, "Emotions matter in crisis: The role of anger and sadness in the publics' response to crisis news framing and corporate crisis response", Communication Research, 38, 6, 2011, pp. 826-855.

[13] J. Kim, R. LaRose, and W. Peng, "Loneliness as the Cause and the Effect of Problematic Internet Use: The Relationship between Internet Use and Psychological WellBeing", CyberPsychology \& Behavior, 12, 4, 2009, pp. 451455 .

[14] A. Lang, "Motivated cognition (LC4MP): The influence of appetitive and aversive activation on the processing of video games", Digital media: Transformation in human communication, 2006, pp. 237-256.

[15] A. Lang, "Using the Limited Capacity Model of Motivated Mediated Message Processing to Design Effective Cancer Communication Messages", Journal of Communication, 56, 2006, pp. S57-S80. 9

[16] A. Lang, R.F. Potter, and P.D. Bolls, Where psychophysiology meets the media: Taking the effects out of media research, in Media effects: Advances in Theory and Research, J. Bryant and M.B. Oliver, Editors. 2009, Routledge: New York. p. 185-206.

[17] A.S. Lee and R.L. Baskerville, "Generalizing generalizability in information systems research", Information systems research, 14, 3, 2003, pp. 221243.

[18] S. Lee and A. Lang, "Discrete emotion and motivation: Relative activation in the appetitive and aversive motivational systems as a function of anger, sadness, fear, and joy during televised information campaigns", Media Psychology, 12, 2, 2009, pp. 148170.

[19] D.T. Lykken and P.H. Venables, "DIRECT MEASUREMENT OF SKIN CONDUCTANCE: A PROPOSAL FOR STANDARDIZATION", Psychophysiology, 8, 5, 1971, pp. 656-672. 
[20] S. Matook, J. Cummings, and H. Bala, "Are You Feeling Lonely? The Impact of Relationship Characteristics and Online Social Network Features on Loneliness", Journal of Management Information Systems, 31, 4, 2015, pp. 278310 .

[21] K.E. Matsa and E. Shearer, "News use across social media platforms 2018", 2018 [cited 2019 5/6/2019]; Available from: https://www.journalism.org/2018/09/10/news-useacrosssocial-media-platforms-2018/.

[22] R.K. Minas, R.F. Potter, A.R. Dennis, V. Bartelt, and S. Bae, "Putting on the Thinking Cap: Using NeuroIS to Understand Information Processing Biases in Virtual Teams", Journal of Management Information Systems, 30, 4, 2014, pp. 49-82.

[23] P. Moravec, R.K. Minas, and A.R. Dennis, "Fake news on social media: People believe what they want to believe when it makes no sense at all", MIS Quarterly, in press.

[24] M. Opgenhaffen and L. d'Haenens, "The impact of online news features on learning from news. A knowledge experiment", International Journal of Internet Science, 6, 1, 2011, pp. 8-28.

[25] R.F. Potter and P.D. Bolls, Psychophysiological measurement and meaning: Cognitive and Emotional Processing of Media, Routledge, New York, 2011.

[26] S. Salim, "How much time do you spend on social media? Research says 142 minutes per day", 2019 [cited 2019 6/8/2019]; Available from: https://www.digitalinformationworld.com/2019/01/h owmuch-time-do-people-spend-social-mediainfographic.html.

[27] R.P. Spicer, S.M. Russell, and E.S. Rosenberg, The mixed reality of things: emerging challenges for human information interaction. SPIE Defense + Security. Vol. 10207. SPIE, 2017.

[28] J.F. Thayer, F. Åhs, M. Fredrikson, J.J. Sollers, and T.D. Wager, "A meta-analysis of heart rate variability and neuroimaging studies: Implications for heart rate variability as a marker of stress and health", Neuroscience \& Biobehavioral Reviews, 36, 2, 2012, pp. 747-756.

[29] G.J. Tortora and B. Derrickson, Principles of anatomy \& physiology, John Wiley \& Sons, Incorporated, 2017.

[30] Y. Tsfati, "Online News Exposure and Trust in the Mainstream Media: Exploring Possible Associations", American Behavioral Scientist, 54, 1, 2010, pp. 22-42.

[31] O. Turel and A. Serenko, "The benefits and dangers of enjoyment with social networking websites", European Journal of Information Systems, 21, 5, 2012, pp. 512-528.

[32] O. Turel, Q. He, G. Xue, L. Xiao, and A. Bechara, "Examination of Neural Systems Sub-Serving Facebook “Addiction”", Psychological Reports, 115, 3, 2014, pp. 675695.

[33] P.H. Vettehen, K. Nuijten, and A. Peeters, "Explaining effects of sensationalism on liking of television news stories: The role of emotional arousal", Communication Research, 35, 3, 2008, pp. 319-338.

[34] S. Vosoughi, D. Roy, and S. Aral, "The spread of true and false news online", Science, 359, 6380, 2018, pp. 11461151.

[35] K. Wise, S. Alhabash, and H. Park, "Emotional responses during social information seeking on Facebook", Cyberpsychology, Behavior, and Social Networking, 13, 5, 2010, pp. 555-562. 
Table 1. Results of HLM analysis

\begin{tabular}{|c|c|c|c|c|c|c|}
\hline & \multicolumn{3}{|c|}{ RSA } & \multicolumn{3}{|c|}{ EDA } \\
\hline & $\boldsymbol{\beta}$ & SE & $t$ & $\beta$ & $S E$ & $t$ \\
\hline \multirow{2}{*}{\multicolumn{7}{|c|}{$\begin{array}{l}\text { Full Model } \\
\text { Intercept }\end{array}$}} \\
\hline & & & & & & \\
\hline Intercept & $5.034 * * *$ & 0.078 & 64.239 & $0.759 * * *$ & 0.018 & 42.437 \\
\hline Time Spent on SM & $0.586 * * *$ & 0.083 & 7.043 & $0.063^{* * *}$ & 0.017 & 3.785 \\
\hline Stay Informed & $-0.237 * *$ & 0.081 & -2.939 & $0.088^{* * *}$ & 0.015 & 6.028 \\
\hline \multicolumn{7}{|c|}{ Believed True Article (Correct Hit) } \\
\hline Intercept & $0.478^{*}$ & 0.231 & 2.074 & $0.160 * *$ & 0.060 & 2.641 \\
\hline Time Spent on SM & -0.322 & 0.248 & -1.3 & -0.055 & 0.058 & -0.951 \\
\hline Stay Informed & -0.148 & 0.25 & -0.591 & $0.106 *$ & 0.106 & 2.171 \\
\hline \multicolumn{7}{|c|}{ Believed Fake Article (False Positive) } \\
\hline Intercept & 0.347 & 0.224 & 1.548 & $0.170 * *$ & 0.053 & 3.217 \\
\hline Time Spent on SM & $-0.489 *$ & 0.241 & -2.029 & -0.084 & 0.052 & -1.610 \\
\hline Stay Informed & -0.134 & 0.247 & -0.544 & $0.106 *$ & 0.048 & 2.214 \\
\hline \multicolumn{7}{|c|}{ Did not believe true article (False Negative) } \\
\hline Intercept & -0.340 & 0.249 & -1.361 & 0.099 & 0.056 & 1.787 \\
\hline Time Spent on SM & 0.206 & 0.297 & 0.695 & -0.069 & 0.057 & -1.224 \\
\hline Stay Informed & -0.340 & 0.271 & -1.899 & 0.075 & 0.047 & 1.595 \\
\hline \multicolumn{7}{|c|}{ Did not believe fake article (Correct Rejection) } \\
\hline Intercept & 0.002 & 0.251 & 0.007 & $0.132^{*}$ & 0.054 & 2.419 \\
\hline Time Spent on SM & $0.582 *$ & 0.294 & 1.979 & -0.033 & 0.059 & -0.558 \\
\hline Stay Informed & $-0.719 * *$ & 0.265 & -2.714 & $0.112^{*}$ & 0.049 & 2.306 \\
\hline \multicolumn{7}{|l|}{ Intent to Share on SM } \\
\hline Intercept & -0.084 & 0.157 & -0.537 & 0.018 & 0.032 & 0.546 \\
\hline Time Spent on SM & 0.108 & 0.123 & 0.884 & -0.040 & 0.026 & -1.512 \\
\hline Stay Informed & $0.326 * *$ & 0.113 & 2.894 & -0.016 & 0.019 & -0.832 \\
\hline \multicolumn{7}{|c|}{ Intent to Share article with their Opinion on SM } \\
\hline Intercept & $-0.486 * *$ & 0.166 & -2.924 & 0.016 & 0.036 & -0.435 \\
\hline Time Spent on SM & -0.158 & 0.14 & -1.129 & $0.111^{* * *}$ & 0.030 & 3.638 \\
\hline Stay Informed & 0.010 & 0.119 & 0.081 & -0.021 & 0.020 & -1.055 \\
\hline
\end{tabular}

